\title{
Correlation between Serum Ferritin Level and Histopathological Disease Severity in Non-alcoholic Fatty Liver Disease
}

\author{
Seyed Reza Modares Mousavi ${ }^{1}$, Bita Geramizadeh ${ }^{2}$, Amir Anushiravani ${ }^{3}$, Fardad Ejtehadi ${ }^{4}$, \\ Mohammad Hossein Anbardar ${ }^{5}$, Maryam Moini 6,*
}

1. Division of Gastroenterology, Department of Internal Medicine, Ahvaz Jundishapur University of Medical Sciences, Ahwaz, Iran

2. Shiraz Transplant Research Center, Shiraz University of Medical Sciences, Shiraz, Iran

3. Digestive Disease Research Institute, Tehran University of Medical Sciences, Tehran, Iran

4. Gastroenterohepatology Research Center, Shiraz University of Medical Sciences, Shiraz, Iran

5. Department of Pathology, Shiraz University of Medical Sciences, Shiraz, Iran

6. Non-communicable Disease Research Center, Shiraz University of Medical Sciences, Shiraz, Iran

* Corresponding Author:

Maryam Moini, MD Non-communicable Disease Research Center, Shiraz University of Medical Sciences, Shiraz, Iran Telefax: + 987136474316 Email: moeenim@sums.ac.ir

Received: 10 Dec. 2017 Accepted: 09 Mar. 2018

\section{ABSTRACT}

\section{BACKGROUND}

Non-alcoholic fatty liver disease (NAFLD) is one of the most common causes of chronic liver disease worldwide. Recently several parameters, such as serum ferritin, have emerged as possible predictors for the severity of NAFLD and insulin sensitivity. We aimed to investigate the value of serum ferritin level as a useful biomarker for the prediction of histopathological disease severity in non-alcoholic steatohepatitis (NASH), the necro-inflammatory form of NAFLD.

\section{METHODS}

This was a prospective cross sectional study in which demographic, clinical, histological, laboratory, and anthropometric data of 30 adult patients with biopsy-proven NAFLD/NASH were analyzed.

\section{RESULTS}

In our patients population with mean age of 37.9 years and mean BMI of 26.5 , statistical analysis did not show a significant difference between the three grades of steatosis in the mean ferritin levels $(p=0.559)$. It was also observed that ferritin level did not have a significant correlation with the stage of fibrosis $(p=0.228)$. The mean transferrin saturation did not show significant difference in different stages and grades of NASH ( $p=0.260$ and 0.944 , respectively), either.

\section{CONCLUSION}

Serum ferritin level may not be useful as a single marker for the prediction of histopathological severity of disease in young patients with NASH who are not morbidly obese.

\section{KEYWORDS:}

Ferritin, Histopathology, Non-alcoholic Fatty Liver Disease, Non-alcoholic Steatohepatitis

Please cite this paper as:

Modares Mousavi SR, Geramizadeh B, Anushiravani A, Ejtehadi F, Anbardar MH, Moini M. Correlation between Serum Ferritin Level and Histopathological Disease Severity in Non-alcoholic Fatty Liver Disease. Middle East J Dig Dis 2018;10:90-95. doi: 10.15171/ mejdd.2018.96.

\section{INTRODUCTION}

Non-alcoholic fatty liver disease (NAFLD) is the $2^{\text {nd }}$ most common cause of cirrhosis and liver transplantation worldwide. It will probably be the leading cause of chronic liver disease in upcoming years. ${ }^{1}$ As the hepatic manifestation of the metabolic syndrome; NAFLD is gaining prevalence worldwide due to the obesity epidemic. ${ }^{2}$ On the other hand, NAFLD could be considered as a multisystem disease that increases the risk of diabetes mellitus, cardiovascular disease, and chronic kidney disease. ${ }^{3}$ 
There is a wide spectrum of liver diseases ranging from simple steatosis to non-alcoholic steatohepatitis (NASH) to cirrhosis and its related complications such as hepatocellular carcinoma and death at the end in NAFLD. ${ }^{4}$ Although the pathogenesis of NAFLD/NASH is not well defined yet, there is a strong evidence for the association of insulin resistance with the development of NAFLD. In progression to NASH, other contributing factors leading to oxidative injury are possibly involved. ${ }^{5}$

The gold standard method for the diagnosis of NASH is liver biopsy, which has its own pitfalls. It is limited by intra and inter-observer variability for pathology interpretation, sampling errors, and invasiveness. ${ }^{6}$ Different scoring systems have been designed, hoping to diagnose and stage NASH without histological data, but the debate on their accuracy is still ongoing. ${ }^{7}$ Transient elastrography is emerging as a non-invasive and reliable tool for diagnosis of fibrosis stages in chronic liver disease, ${ }^{8}$ with the ability of grading steatosis using controlled attenuated parameter, but it could not be a substitute for liver biopsy for the diagnosis of NASH.

Recently, several non-invasive markers such as serum ferritin have emerged as possible predictors for the presence of NASH versus simple steatosis. ${ }^{9} 10$ Iron overload is found in as many as one third of the patients with NAFLD. ${ }^{11}$ Steatosis, insulin resistance, and inflammation cause an altered regulation of iron transport. ${ }^{12}$

Ferritin is an acute phase reactant; however, its elevation in the serum of patients with fatty liver disease may not only be a sign of disease activity. The role of altered iron metabolism in the development of NAFLD and disease progression has been a subject of interest for investigation in many studies. A variety of pathogenesis mechanisms have been defined for iron including production of reactive oxygen species, disturbed insulin signaling, and altered lipid metabolism in hepatic tissue and their resultant damage. ${ }^{13}$ Elevated serum ferritin has been found to be correlated with hepcidine level, which is an iron regulatory hormone, and also with the level of hepatic iron in patients with NAFLD, which may point to the pathological role of iron metabolism in such patients. ${ }^{14}$ However, despite all these, there is no general agreement for the correlation between serum ferritin level and disease severity in NASH in different studies. In the present study, we aimed to investigate the usefulness of evaluating serum ferritin level for the prediction of histological disease severity in NASH in our patients.

\section{MATERIALS AND METHODS}

In the present cross sectional prospective study, demographic, clinical, histological, laboratory, and anthropometric data of 30 adult patients with biopsyproven NAFLD/NASH were analyzed.

The Ethics Committee of Shiraz University of Medical Sciences approved this study (which was part of a thesis) and a written informed consent was obtained from each patient to take part in the study.

The patients were selected form those who referred to outpatient subspecial clinics of gastroenterology and hepatology affiliated to Shiraz University of Medical Science for evaluation of abnormal liver tests, for whom liver biopsy had been requested by the responsible hepatologist for confirmation of diagnosis or evaluation of the disease severity.

Liver biopsy was performed under the guide of ultrasonography by an expert radiologist and all samples were sent to the pathology lab and interpreted by a single hepatopathologist.

Diagnosis of fatty liver disease was made if large fat vacuoles were present in the liver parenchyma, displacing the nuclei to the border of the cells. Steatosis was graded as 1 if less than $33 \%$ of the hepatocytes were affected, grade 2 when $33-66 \%$ of the hepatocytes were affected, and grade 3 if more than $66 \%$ of the hepatocytes were affected. Staging and grading were performed according to the Brunt et al scoring. ${ }^{15}$

Serum ferritin was measured by ELISA method (Monobind, USA) within two weeks after performing the liver biopsy. Serum iron and total iron-binding capacity (TIBC) were measured by colorimetric methods at the same time. Transferrin saturation was calculated as the percentage of serum iron to TIBC ratio.

The patients who had the following criteria were excluded: chronic liver disease with other etiologies rather than NAFLD (such as viral hepatitis B or C, autoimmune hepatitis, Wilson's disease, hemochromatosis), alcohol consumption $>20 \mathrm{~g} /$ day, using iron supplements, presence of any kind of infection, or history of rheumatologic disorders. The patients who were taking medications known to cause fatty liver disease such as steroids, methotrexate, 
Table 1: Demographic data and biochemical laboratory values of patients with non-alcoholic fatty liver disease according to the grade of steatohepatitis

\begin{tabular}{lccccc}
\hline Characteristic & All Patients (30) & No Steatohepatitis (5) & $\begin{array}{c}\text { Mild } \\
\text { Steatohepatitis (19) }\end{array}$ & $\begin{array}{c}\text { Moderate } \\
\text { Steatohepatitis (4) }\end{array}$ & $\begin{array}{c}\text { Severe } \\
\text { Steatohepatitis (2) }\end{array}$ \\
\hline Age mean (years) & $37.93 \pm 12.5$ & $34.8 \pm 14.8$ & $39.15 \pm 12.7$ & $32.75 \pm 7.4$ & $44.5 \pm 17.6$ \\
\hline Male sex (n, \%) & $17(56.7 \%)$ & $1(20 \%)$ & $13(68.4 \%)$ & $3(75 \%)$ & 0 \\
\hline BMI mean (kg/m²) & $26.45 \pm 4.4$ & $25.3 \pm 8.3$ & $26.8 \pm 3.7$ & $25.0 \pm 1.8$ & $29.0 \pm 1.4$ \\
\hline AST (IU/L) & $54.8 \pm 37.7$ & $31.6 \pm 20.7$ & $49.8 \pm 25.5$ & $79.2 \pm 34.7$ & $112.0 \pm 110$ \\
\hline ALT (IU/L) & $85.7 \pm 57.1$ & $46.2 \pm 29.3$ & $79.1 \pm 37.9$ & $134.3 \pm 92.2$ & $151.0 \pm 115.9$ \\
\hline ALP (IU/L) & $229.4 \pm 98.7$ & $338.4 \pm 154.9$ & $205.6 \pm 75.8$ & $204.5 \pm 29.7$ & $233.5 \pm 89.8$ \\
\hline Serum Ferritin (ng/mL) & $200.8 \pm 200.6$ & $88.0 \pm 106$ & $212.1 \pm 236$ & $268.5 \pm 61.8$ & $241.6 \pm 146.2$ \\
\hline $\begin{array}{l}\text { Fasting blood sugar } \\
\text { (mg/dL) }\end{array}$ & $115.0 \pm 76.7$ & $94.8 \pm 13$ & $95.3 \pm 26.1$ & $179 \pm 153.3$ & $225.5 \pm 193$ \\
\hline
\end{tabular}

ALP: Alkaline phosphatase, ALT: alanine transferase, AST: aspartate transferase, BMI: body mass index

Table 2: Pearson correlation coefficient between mean serum ferritin levels and different variables in patients with non-alcoholic fatty liver disease

\begin{tabular}{lcc}
\hline Variable & Pearson correlation coefficient & P value \\
\hline Age & 0.296 & 0.112 \\
\hline BMI & -0.032 & 0.869 \\
\hline AST & 0.039 & 0.839 \\
\hline ALT & 0.096 & 0.615 \\
\hline ALP & -0.351 & 0.057 \\
\hline Transferrin saturation & 0.312 & 0.093 \\
\hline Cholesterol & -0.150 & 0.429 \\
\hline Triglyceride & -0.160 & 0.399 \\
\hline Fasting plasma glucose & 0.057 & 0.763 \\
\hline
\end{tabular}

ALP: Alkaline phosphatase, ALT: alanine transferase, AST: aspartate transferase, BMI: body mass index

and tamoxifen were also excluded. Those who had already received medical treatment for fatty liver disease at the time of study were excluded, too.

Demographic data such as age, sex, rural or urban residence, medical history, and medications were obtained through interviews with the patients. A physical examination including measuring blood pressure, body weight, and height measures was performed. Other laboratory tests including hepatic, hematologic, metabolic, and lipid profile tests were performed.

We used a One-Sample Kolmogorov-Smirnov test to evaluate normal distribution of our variables. One-way ANOVA, and Pearson's Correlation Coefficient were used to determine the relation between mean serum ferritin levels and other variables. Independent sample $t$ test was used to compare the means. All analyses were performed using Statistical Package for Social Sciences (SPSS, Chicago, IL, USA) software version 16.0 for windows. $P$ values less than 0.05 were considered to be statistically significant.

\section{RESULTS}

A total of 30 patients with biopsy-proven NAFLD with mean age of 37.9 years and mean BMI of 26.5 were recruited in this study. Demographic data and mean biochemical values are shown in table 1.

Of the studied patients, 16 had mild and 14 had moderate macrovesicular steatosis. Regarding the fibrosis stage; there were $13(43.3 \%)$ patients in stage 0 , three $(10 \%)$ in stage 1 , eight $(26.7 \%)$ in stage 2 , four $(13.3 \%)$ in stage 3, and two (6.7\%) patients in stage 4 . Regarding the steatohepatitis grades; there were 19 patients $(63.3 \%)$ with mild, four (13.3\%) with moderate, and two (6.7\%) patients with severe steatohepatitis while five (16.7\%) patients had no steatohepatitis.

Eleven (36.7\%) patients had ferritin levels above 200 $\mathrm{ng} / \mathrm{L}$ and only six patients had transferrin saturation more than $45 \%$. As it was expected, men had significantly higher ferritin levels than women $(280.08 \pm 222.92$ vs. $97.27 \pm$ $102.77, p=0.011$ ). The correlation between mean serum level of ferritin and different variables is shown in table 2. 


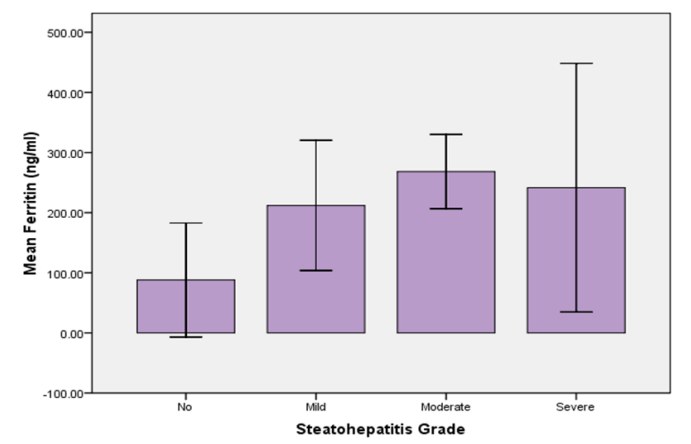

Fig.1: Comparison of the mean serum ferritin levels between different grades of steatohepatitis in liver biopsy

There was no correlation between serum ferritin level and age, body mass index (BMI), the level of liver enzymes, fasting plasma sugar, or serum lipids.

Figure 1 shows the relation between the grades of steatohepatitis in liver biopsy and serum ferritin levels. One-way ANOVA did not show a significant difference between these three grades of steatohepatitis in mean ferritin levels $(p=0.559)$. It was also observed that ferritin levels did not have a significant correlation with the stage of fibrosis in liver histological evaluation of our patients ( $p=0.228$, figure 2 ). It was also observed that although mean serum ferritin level was lower in patients without steatohepatitis comparing with those with steatohepatitis ( 88 vs. $223.4 \mathrm{ng} / \mathrm{mL}$ ), this difference was not statistically significant. This was also true for the mean transferrin saturation, which did not show significant difference in different stages and grades of NASH in liver histology ( $p=0.260$, and 0.944 , respectively).

\section{DISCUSSION}

We conducted a prospective cross sectional study on 30 patients with biopsy proven NAFLD/NASH and found no significant correlation between the histopathological stages of the disease with serum ferritin levels. The prevalence of NAFLD and its severity increase with age. Our patients were relatively young and with less severe disease as expected.

Several studies have reported elevated serum ferritin levels in patients with NAFLD. ${ }^{16,17}$ High ferritin level has been regarded as a marker of more severe histological derangement of liver in NASH rather than true iron overload. ${ }^{18}$

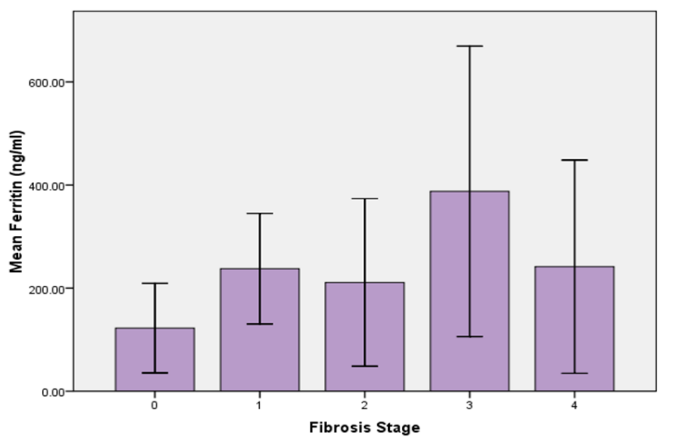

Fig.2: Comparison of the mean of serum ferritin levels between different stages of liver fibrosis in patients with non-alcoholic fatty liver disease

Kowdley and colleagues showed an independent association between serum ferritin level and increased risk of advanced fibrosis in NAFLD. They proposed that levels $>1.5 \times$ upper normal limits can have a predictive value for liver fibrosis in their patients with severe obesity and metabolic syndrome. ${ }^{19}$ Serum ferritin levels and BMI were strongly associated with fibrosis, and portal and lobular inflammation in patients with NAFLD, as Manousou and co-workers reported, in patients with biopsy proven NAFLD. ${ }^{20}$

On the other hand, in a retrospective study by Angulo and others on over 1400 patients with NAFLD, ferritin did not correlate with any grade of liver fibrosis and the accuracy of non-invasive fibrosis scoring systems did not improve with the inclusion of serum ferritin. They concluded that serum ferritin had a low level of accuracy for diagnosis and staging of NAFLD. ${ }^{21}$

Yoneda and colleagues showed similar results after analyzing the data of 1200 patients with biopsy proven NAFLD, which belonged to a large Japanese cohort database. They showed that serum ferritin level was independently associated with steatosis grade and fibrosis stage, but it was inaccurate as a predictive test of liver fibrosis, because several other factors including sex and metabolic features could have interfered. Their conclusion was that serum ferritin had a low diagnostic accuracy for detecting fibrosis in patients with NAFLD when considered alone. $^{22}$

In our study, no significant correlation between serum ferritin level and histopathological grade or stage of the disease was seen. This may be partly due to limitations in our study. We had only 30 patients with biopsy proven 
NAFLD/NASH. Our patients were relatively young with a mean age of 37.9 years, which may affect the results. There is the possibility of the presence of true iron deficiency anemia especially in young women, which interferes with the interpretation of the results of iron profile. On the other hand our patients were not severely obese (mean BMI: 26.5). Obesity and metabolic features are among factors that may affect the iron profile.

Currently, the diagnosis of NAFLD is usually made using non-invasive methods and there is low desire for clinicians to refer the patients with probable diagnosis of NAFLD/NASH for liver biopsy. Such referrals are usually seen in cases when other causes of liver disease could not be ruled out or there is unsatisfactory response to treatment. We regard this as the main reason for limited number of cases in our prospective study. The limitations for performing liver biopsy are even more in elderly patients while comorbidities and using anti platelet medications are more prevalent, putting them at higher risk of complications. This may also be a reason for the limited number of our patients and their relatively younger age.

\section{CONCLUSION}

Regarding our study results and lack of consensus between different studies, 6,11,13,14,20,22,23 it can be concluded that serum ferritin level may not be a single reliable marker of disease severity in young patients with NAFLD who are not morbidly obese.

\section{ETHICAL APPROVAL}

There is nothing to be declared.

\section{CONFLICT OF INTEREST}

The authors declare no conflict of interest related to this work.

\section{REFERENCES}

1. Wong RJ, Aguilar M, Cheung R, Perumpail RB, Harrison SA, Younossi ZM, et al. Nonalcoholic steatohepatitis is the second leading etiology of liver disease among adults awaiting liver transplantation in the United States. Gastroenterology 2015;148:547-55. doi: 10.1053/j.gastro.2014.11.039.

2. Zezos P, Renner EL. Liver transplantation and nonalcoholic fatty liver disease. World $J$ gastroenterol 2014;20:15532-8. doi: 10.3748/wjg.v20.i42.15532.

3. Byrne CD, Targher G. NAFLD: a multisystem disease.
J Hepatol 2015;62(1 Suppl):S47-64. doi: 10.1016/j. jhep.2014.12.012.

4. Serfaty L, Lemoine M. Definition and natural history of metabolic steatosis: clinical aspects of NAFLD, NASH and cirrhosis. Diabetes Metab 2008;34:634-7. doi: 10.1016/S1262-3636(08)74597-X.

5. Magee N, Zou A, Zhang Y. Pathogenesis of Nonalcoholic Steatohepatitis: Interactions between Liver Parenchymal and Nonparenchymal Cells. Biomed Res Int 2016;2016:5170402. doi: 10.1155/2016/5170402.

6. Lombardi R, Pisano G, Fargion S. Role of Serum Uric Acid and Ferritin in the Development and Progression of NAFLD. Int J Mol Sci 2016;17:548. doi:10.3390/ ijms17040548.

7. Buzzetti E, Lombardi R, De Luca L, Tsochatzis EA. Noninvasive Assessment of Fibrosis in Patients with Nonalcoholic Fatty Liver Disease. Int $J$ Endocrinol 2015;2015:343828. doi: 10.1155/2015/343828.

8. Tsochatzis EA, Gurusamy KS, Ntaoula S, Cholongitas E, Davidson BR, Burroughs AK. Elastography for the diagnosis of severity of fibrosis in chronic liver disease: a meta-analysis of diagnostic accuracy. $J$ Hepatol 2011;54:650-9. doi: 10.1016/j.jhep.2010.07.033.

9. Feldstein AE, Wieckowska A, Lopez AR, Liu YC, Zein $\mathrm{NN}$, McCullough AJ. Cytokeratin-18 fragment levels as noninvasive biomarkers for nonalcoholic steatohepatitis: a multicenter validation study. Hepatology 2009;50:10728. doi: 10.1002/hep.23050.

10. Sumida $\mathrm{Y}$, Yoneda M, Hyogo $\mathrm{H}$, Yamaguchi $\mathrm{K}$, Ono M, Fujii $\mathrm{H}$, et al. A simple clinical scoring system using ferritin, fasting insulin, and type IV collagen $7 \mathrm{~S}$ for predicting steatohepatitis in nonalcoholic fatty liver disease. J Gastroenterol 2011;46:257-68. doi: 10.1007/s00535-010-0305-6.

11. Datz C, Muller E, Aigner E. Iron overload and nonalcoholic fatty liver disease. Minerva Endocrinol 2017;42:173-83. doi: 10.23736/S0391-1977.16.02565-7.

12. Dongiovanni P, Fracanzani AL, Fargion S, Valenti L. Iron in fatty liver and in the metabolic syndrome: a promising therapeutic target. $J$ Hepatol 2011;55:920-32. doi: 10.1016/j.jhep.2011.05.008.

13. Nelson JE, Klintworth H, Kowdley KV. Iron metabolism in Nonalcoholic Fatty Liver Disease. Curr Gastroenterol Rep 2012;14:8-16. doi: 10.1007/s11894-011-0234-4.

14. Ryan JD, Armitage AE, Cobbold JF, Banerjee R, Borsani $\mathrm{O}$, Dongiovanni P, et al. Hepatic iron is the major determinant of serum ferritin in NAFLD patients. Liver Int 2018;38:164-73. doi: 10.1111/liv.13513.

15. Brunt EM, Janney CG, Di Bisceglie AM, NeuschwanderTetri BA, Bacon BR. Nonalcoholic steatohepatitis: a proposal for grading and staging the histological lesions. $\mathrm{Am}$ J Gastroenterol 1999;94:2467-74. doi: 10.1111/j.15720241.1999.01377.x.

16. Brudevold R, Hole T, Hammerstrom J. Hyperferritinemia is associated with insulin resistance and fatty liver in patients without iron overload. PloS one 2008;3:e3547. doi: 
10.1371/journal.pone.0003547.

17. Fargion S, Mattioli M, Fracanzani AL, Sampietro M, Tavazzi D, Fociani P, et al. Hyperferritinemia, iron overload, and multiple metabolic alterations identify patients at risk for nonalcoholic steatohepatitis. Am J Gastroenterol 2001;96:2448-55. doi: 10.1111/j.1572-0241.2001.04052.x.

18. Bugianesi E, Manzini P, D’Antico S, Vanni E, Longo F, Leone N, et al. Relative contribution of iron burden, HFE mutations, and insulin resistance to fibrosis in nonalcoholic fatty liver. Hepatology 2004;39:179-87. doi: 10.1002/hep.20023.

19. Kowdley KV, BeltP, Wilson LA, Yeh MM, NeuschwanderTetri BA, Chalasani N, et al. Serum ferritin is an independent predictor of histologic severity and advanced fibrosis in patients with nonalcoholic fatty liver disease. Hepatology 2012;55:77-85. doi: 10.1002/hep.24706.

20. Manousou P, Kalambokis G, Grillo F, Watkins J, Xirouchakis E, Pleguezuelo M, et al. Serum ferritin is a discriminant marker for both fibrosis and inflammation in histologically proven non-alcoholic fatty liver disease patients. Liver Int 2011;31:730-9. doi: 10.1111/j.14783231.2011.02488.x.

21. Angulo P, George J, Day CP, Vanni E, Russell L, De la Cruz AC, et al. Serum ferritin levels lack diagnostic accuracy for liver fibrosis in patients with nonalcoholic fatty liver disease. Clin Gastroenterol Hepatol 2014;12:11639.e1. doi: 10.1016/j.cgh.2013.11.035.

22. Yoneda M, Thomas E, Sumida Y, Imajo K, Eguchi Y, Hyogo H, et al. Clinical usage of serum ferritin to assess liver fibrosis in patients with non-alcoholic fatty liver disease: Proceed with caution . Hepatol Res 2014;44:499502. doi: 10.1111/hepr.12327.

23. Zhao L, Li T, Wang Y, Pan Y, Ning H, Hui X, et al. Elevated plasma osteopontin level is predictive of cirrhosis in patients with hepatitis B infection. Int J Clin Pract 2008;62:1056-62. doi: 10.1111/j.1742-1241.2007.01368.x. 